\title{
ANÁLISE DE DANOS DE COLHEITA DE MADEIRA EM FLORESTA TROPICAL ÚMIDA SOB REGIME DE MANEJO FLORESTAL SUSTENTADO NA AMAZÔNIA OCIDENTAL ${ }^{1}$
}

\author{
Alberto Carlos Martins Pinto², Agostinho Lopes de Souza ${ }^{3}$, Amaury Paulo de Souza ${ }^{3}$, Carlos Cardoso Machado \\ Luciano José Minette ${ }^{4}$ e Antonio Bartolomeu do Vale ${ }^{3}$
}

\begin{abstract}
RESUMO - Procurou-se identificar, qualificar e quantificar a intensidade de danos causados pela colheita florestal em uma área com floresta primária explorada $(\boldsymbol{F P E})$, tendo com testemunha uma área com floresta primária nãoexplorada (FPNE), com 202 e 204 ha, respectivamente. Na $\boldsymbol{F P N E}$, foram avaliados os danos decorrentes de causas naturais. As áreas de $\boldsymbol{F P E}$ e de $\boldsymbol{F P N E}$ localizam-se no município de Manicoré, Estado do Amazonas. Realizou-se o inventário utilizando o método de amostragem aleatória, em ambas as áreas. As amostragens foram executadas em dois níveis de inclusão de $\boldsymbol{D A P}$. No nível I foram instaladas cinco parcelas de $100 \times 100 \mathrm{~m}$ (1 ha), onde foram inventariadas todas as árvores com $\boldsymbol{D A P} \geq 15 \mathrm{~cm}$. No nível II, as parcelas do nível I foram divididas sistematicamente em subparcelas de $10 \times 10 \mathrm{~m}\left(100 \mathrm{~m}^{2}\right)$, tendo sido amostradas aleatoriamente cinco subparcelas por parcela do nível I, totalizando $2.500 \mathrm{~m}^{2}$, onde foram inventariados todos os indivíduos (varejões) com 5,0 $\leq \boldsymbol{D A P}<15 \mathrm{~cm}$. As análises dos danos decorrentes de causas naturais indicaram que na $\boldsymbol{F P N E}$ apenas $10 \%$ da vegetação adulta remanescente apresentou danos. Na $\boldsymbol{F P E}$, os danos causados pelas operações de colheita florestal, incluindo-se o corte e o arraste de toras, totalizaram $29 \%$. As modificações na estrutura diamétrica da $\boldsymbol{F P E}$ foram maiores nas maiores classes de $\boldsymbol{D} \boldsymbol{A P}$, em que, em geral, foram constatadas reduções de 27, 29 e 30\%, respectivamente, no número de árvores (n/ha), na área basal $\left(\mathrm{m}^{2} / \mathrm{ha}\right)$ e no volume $\left(\mathrm{m}^{3} / \mathrm{ha}\right)$. Essa redução de estoque foi considerada normal, uma vez que a amostragem foi realizada imediatamente após a execução das operações de colheita florestal.
\end{abstract}

Palavras-chave: Floresta tropical, colheita florestal e danos.

\section{ANALYSIS OF LOGGING DAMAGE IN A MOIST TROPICAL FOREST UNDER SUSTAINED MANAGEMENT IN WESTERN AMAZON}

\begin{abstract}
The objective of this work was to identify, qualify and quantify the intensity of damage from natural causes and forest harvest in a non-exploited old growth forest (FPNE) and in a harvested old growth forest (FPE). The studied area is located in the counties of Manicoré, Amazon, Brazil, with an area of 204 ha of FPNE and 202 ha of FPE, respectively. The inventory was carried out applying the randomized sampling method, in both areas. Sampling collection was carried out in two levels of approach. In level I, five plots of $100 \times 100 \mathrm{~m}$ (1 ha) were established, and the individuals with $D B H \geq 15 \mathrm{~cm}$ were evaluated. In level II, the plots of level I were systematically subdivided in subplots of $10 \times 10 \mathrm{~m}\left(100 \mathrm{~m}^{2}\right)$, being randomly sampled only five of these per plot of level I, with an area of 2,500 $\mathrm{m}^{2}$, where all the individuals between $5.0 \leq \mathrm{DBH}<15 \mathrm{~cm}$ (large samplings) were evaluated. The assessment of damage caused to the remaining trees indicate that only $10 \%$ of the adult vegetation presented damages in the non-exploited forest. In the exploited forest, the damage caused by harvest operations, such as felling log extraction, skidding trails and clearcut, was of 29\%. The changes in the structures of volume, basal area and diameter were more evident in the greater diameter classes, where reductions of 30\%, 29\% and $27 \%$, occurred respectively. Nevertheless, this reduction can be considered normal, since the sampling was collected immediately after forest harvest operations.
\end{abstract}

Key words: Tropical forest, forest harvest, damages.

1 Recebido para publicação em 20.7.2001.

Aceito para publicação em 20.6.2002.

2 Eng. Florestal, M.S. em Ciência Florestal, DEF/UFV; bolsista da CAPES, <amartins@inpa.gov.br>; ${ }^{3}$ Professores do Dep. de Engenharia Florestal da Universidade Federal de Viçosa - UFV, 36571-000 Viçosa-MG; ${ }^{4}$ Pesquisador do Dep. de Engenharia Florestal da UFV. 


\section{INTRODUÇÃO}

Embora o manejo florestal precise ser executado de forma a manter, no mínimo, a sustentabilidade ambiental, observa-se que os produtos de base florestal natural que circulam no mercado não procedem de florestas manejadas. Nos últimos anos, a Floresta Amazônica tem merecido atenção especial, pelo fato de essa formação conter a maior reserva de recursos florestais e ser depositária da maior biodiversidade do Planeta. Entretanto, seus recursos madeireiros e não-madeireiros estão sendo explorados de forma irracional, uma vez que predomina a colheita madeireira sem o mínimo planejamento. Essa colheita é caracterizada pela máxima retirada de madeira por unidade de área, das espécies de valor comercial, promovendo danos irreversíveis à floresta remanescente.

A colheita florestal sem planejamento, realizada de maneira intensa e seletiva na Amazônia, tem transformado florestas de elevado estoque de madeira e valor comercial em florestas degradadas, de baixo valor comercial e de difícil recuperação.

A derrubada de árvores praticada na Amazônia é considerada 80\% ilegal (Greenpeace, 1999). Na maior parte dos casos, os Planos de Manejo Florestal não são seguidos, mas usados meramente para satisfazer requerimentos legais. Grande parte da extração considerada "legal" é altamente destrutiva, pois emprega tecnologias de colheita e processamento inadequadas, acarretando enormes danos e desperdícios. Em média, apenas um terço da madeira extraída é transformada em produtos finais (Uhl et al., 1996).

Uma colheita florestal planejada e executada com rigorosos critérios técnicos não só causa baixo impacto ambiental nos meios físico, biótico e antrópico, como também proporciona significativa redução nos custos totais da colheita de madeira. Por conseguinte, contribui para a sustentabilidade ambiental, econômica e social do plano de manejo florestal. Entretanto, a colheita de madeira baseada nas recomendações de um plano de manejo sustentável, por si só, não garante a sustentabilidade da floresta explorada. É importante conhecer a composição florística e as estruturas fitossociológicas e paramétricas da floresta para fundamentar ambientalmente o manejo. Contudo, é necessário ainda um planejamento adequado para prever a intensidade com que os danos da colheita de madeira irão ocorrer nas estruturas e na arquitetura da floresta, a fim de garantir a sustentabilidade ambiental do manejo. O planejamento deve contemplar as técnicas e os métodos de corte, de extração e de transporte mais adequados, no sentido de conservar de impactar, o mínimo possível a estrutura e a arquitetura da floresta.

De acordo com o exposto, os objetivos do presente trabalho foram identificar, qualificar e quantificar a intensidade de danos à estrututura das florestas primárias nãoexplorada e explorada, causados pela colheita florestal, em floresta primária explorada, em termos de volume, área basal ou dominância absoluta e número de indivíduos por hectare ou densidade absoluta, por nível de inclusão.

\section{MATERIAL E MÉTODOS}

\subsection{Amostragem, Coleta e Análise de Dados}

Foi feito um inventário florestal por amostragem aleatória, com parcelas de área fixa, em áreas independentes de floresta primária não-explorada $(\boldsymbol{F P N E})$ e de floresta primária explorada (FPE), com 204 e 202 ha, respectivamente. As áreas localizam-se no município de Manicoré ( $5^{\circ} 50^{\prime}$ de latitude sul e $61^{\circ} 18^{\prime} 30^{\prime \prime}$ de longitude a oeste de Greenwich), Estado do Amazonas (Otto, 1996). Segundo a classificação de Köppen, o clima predominante é o do tipo Af (tropical chuvoso), com temperaturas médias anuais em torno de 24 e $26^{\circ} \mathrm{C}$ e umidade relativa elevada, variando de 85 a $90 \%$ (Brasil, 1978). O relevo nas áreas de $\boldsymbol{F P N E}$ e de $\boldsymbol{F P E}$ varia de plano a suavemente ondulado. A cobertura vegetal da área está inserida na "Região da Floresta Ombrófila Densa" (IBGE, 1993).

A amostragem foi executada em dois níveis de inclusão de $\boldsymbol{D A P}$, conforme metodologia recomendada por Silva \& Lopes (1984). No nível I, foram instaladas cinco parcelas de $100 \times 100 \mathrm{~m}$ (1 ha) e inventariadas todas as árvores com $\boldsymbol{D A P} \geq 15,0 \mathrm{~cm}$, identificando-se as seguintes características: a) nome vulgar regional das espécies; b) circunferência à altura do peito $(\boldsymbol{C A P})$, em centímetros; c) altura total $(\boldsymbol{H t})$ e altura comercial $(\boldsymbol{H c})$, em metros; a posição de danos $(\boldsymbol{P d})$, em que $\boldsymbol{P} \boldsymbol{d}_{1}=$ sem danos, $\boldsymbol{P} \boldsymbol{d}_{2}=$ danos no tronco, $\boldsymbol{P d}_{3}=$ danos na copa, $\boldsymbol{P} \boldsymbol{d}_{4}$ $=$ danos no tronco e na copa e $\boldsymbol{P d}_{5}=$ árvore morta; e a causa de danos $(\boldsymbol{C} \boldsymbol{d})$, em que $\boldsymbol{C} \boldsymbol{d}_{\mathbf{1}}=\boldsymbol{P} \boldsymbol{d}_{\mathbf{1}}=$ sem danos, $\boldsymbol{C d}_{\mathbf{2}}=$ danos causados pelas operações de corte, $\boldsymbol{C d}_{\mathbf{3}}=$ danos causados pelas operações de extração e $\boldsymbol{C d}_{4}=$ árvore morta pelas operações de colheita florestal. No nível II, cada parcela do nível I foi dividida sistematicamente em 100 subparcelas de $10 \times 10 \mathrm{~m}$, tendo sido amostradas aleatoriamente cinco subparcelas. Em 
cada subparcela foram inventariados todos os indivíduos (varejões) entre 5,0 $\leq \boldsymbol{D A} \boldsymbol{P}<15,0 \mathrm{~cm}$, tendo sido identificadas as mesmas características observadas no nível I.

O delineamento experimental utilizado foi o inteiramente casualizado, nos seguintes arranjos fatoriais: 2 x 5 (florestas e posição de danos); e 2 x 4 (florestas e causa de danos). Os dados de densidade absoluta (DA), dominância absoluta (DoA) e volume por hectare (Vol) foram submetidos às análises de variância (ANOVA), e nos casos em que foram constatadas diferenças significativas entre os fatores estudados as médias foram discriminadas pelo teste de Tukey, a $5 \%$ de probabilidade. Para as variáveis em estudo, foram aplicados os testes de normalidade de Lilliefors e de homogeneidade das variâncias de Cochran e Barllet. As análises estatísticas foram realizadas por meio do programa SAEG (Sistema para Análises Estatísticas), desenvolvido pela Universidade Federal de Viçosa.

\subsection{Caracterização do Sistema de Colheita Florestal}

O sistema de colheita utilizado pela empresa é o de toras longas (Minette, 1996), ou seja, caracterizou-se pela execução do desgalhamento no local de corte e pelas demais operações após a remoção da madeira realizadas nos pátios de embarque ou na beira da estrada. Dois anos antes da colheita, foi executado um mapeamento detalhado de toda a área, que culminou com um inventário a $100 \%$ e mapeamento de todas as árvores com diâmetro à altura do peito $(\boldsymbol{D} A \boldsymbol{P})$ igual ou maior que $50,0 \mathrm{~cm}$. Inclusive, foi utilizado o Sistema de Posicionamento Geográfico (GPS) e foram anotadas as características topográficas locais e de cada talhão. Cada árvore foi cadastrada em um banco de dados, registrando-se a espécie, o $\boldsymbol{D A P}$, a $\boldsymbol{H c}$, a localização, a inclinação do tronco e o destino (colheita, preservação, matriz, defeituosa, $2^{\mathrm{a}}$ colheita etc.). Os dados obtidos do mapeamento e do inventário a $100 \%$ foram analisados por meio do Sistema de Informações Geográficas (SIG) e, então, elaborados mapas temáticos, que foram as bases sobre as quais o planejamento e a execução das atividades de colheita foram fundamentados.

Com o mapa da colheita, a equipe de corte, composta por um técnico, um motosserrista e um ajudante, fazia o reconhecimento da área e marcava as árvores a serem colhidas com um $\mathbf{X}$ e com uma seta, indicando a direção de queda.
O corte florestal incluiu: corte ou derrubada, desgalhamento, destopamento, medição, marcação e préextração (Minette, 1996). Com a localização das árvores a serem colhidas, um técnico demarcou, no campo, os pátios de embarque (principais), os pátios intermediários e as trilhas de arraste. Os pátios de embarque foram localizados nas áreas de melhor acesso, como margem de rios ou de igarapés, pois o transporte seria feito por balsas.

Em seguida, foi repassado para a equipe de corte o mapa de localização das árvores a serem derrubadas, cuja operação de corte incluía limpeza do tronco e teste de oco, que consistia na introdução do sabre da motosserra no tronco no sentido vertical. O corte foi executado, no mínimo, a $10 \mathrm{~cm}$ e, no máximo, a $30 \mathrm{~cm}$ de altura do nível do solo. Quando necessário, a queda foi direcionada com o uso de cunhas. Independentemente do comprimento do tronco, para eliminação da copa, o corte era executado na primeira bifurcação. A plaqueta com o número de identificação da árvore era retirada antes do corte e recolocada no toco, sendo a tora numerada com o número correspondente ao da plaqueta.

As trilhas de arraste foram identificadas por fitas de plástico: uma cor sinalizava as trilhas principais e outra sinalizava as trilhas secundárias. A equipe de abertura das trilhas foi composta por um técnico, um tratorista e um ajudante. As trilhas principais e os pátios foram planejados de forma que o arraste não ultrapassasse a distância de $250 \mathrm{~m}$.

O arraste foi executado com um trator Skidder de pneus. A equipe de arraste foi composta por dois técnicos, dois operadores de Skidders e dois ajudantes. A numeração afixada na tora era retirada antes do arraste, pelo ajudante, e fixada novamente na tora, no momento do embarque.

O transporte das toras até a balsa foi realizado por carretas com cambão telescópico, auxiliado por um carregador frontal, que empilhou as toras na balsa. A balsa tinha dimensões de $10 \mathrm{~m}$ de largura por $30 \mathrm{~m}$ de comprimento. As toras foram dispostas perpendicularmente ao comprimento da balsa.

Durante as operações de colheita foram utilizadas as seguintes máquinas e equipamentos: dois Skidders da marca Müller; um trator de esteiras modelo D4 da marca Caterpillar; quatro motosserras da marca STIHL 051; dois grupos geradores; um barco rebocador; uma voadeira; um barco para transporte da equipe de trabalho; uma balsa para transporte das toras; uma balsa para transporte 
das máquinas pesadas; e capacetes com viseiras e protetor auricular, botas, terçados (facão), cunha e marreta.

\section{RESULTADOS E DISCUSSÃO}

Na floresta primária explorada $(\boldsymbol{F P E})$, os danos à vegetação adulta remanescente $(\boldsymbol{D} \boldsymbol{A P} \geq 15 \mathrm{~cm})$, decorrentes das operações de colheita florestal, foram de 33 (10\%) árvores danificadas por hectare, sendo: $9(3 \%)$ com danos ao tronco, 13 (4\%) com danos à copa, 3 (1\%) com danos no tronco e na copa e 8 (2\%) árvores mortas. $\mathrm{O}$ volume de madeira colhido foi de $18 \mathrm{~m}^{3} / \mathrm{ha}$. Resultados semelhantes foram obtidos por Jonkers (1987), que em um estudo no Suriname destacou que se a colheita de madeira for menor que $20 \mathrm{~m}^{3} / \mathrm{ha}$, raramente, resultará em danos severos à vegetação. Esses resultados estão em consonância com Lanly (1982), que relatou que as taxas de extração de madeira são geralmente baixas e que a colheita em si, se bem planejada, não representa uma ameaça para a produção contínua de madeira. Todavia, qualquer dano decorrente da colheita afeta a produção futura de madeira, e quanto maior os volumes de madeira colhidos, maiores e mais severos serão os danos (Hendrison, 1989).

Do total de árvores danificadas, 14 (43\%) foram árvores de valor comercial, 10 (30\%) foram árvores com potencial futuro e as demais $9(27 \%)$ foram árvores sem valor comercial definido. Resultados muito diferentes foram encontrados por Jonkers (1987), que em $15 \mathrm{~m}^{3} / \mathrm{ha}$ de volume explorado no Suriname obteve apenas 18\% de árvores de valor comercial danificadas.
De modo geral, o porcentual de danos observado na área de estudo foi baixo, se comparado com os resultados obtidos por Veríssimo et al. (1996a, b), na Tailândia e em Paragominas, no sul do Pará, onde foram colhidos, em média, 16 e $38 \mathrm{~m}^{3} /$ ha, respectivamente, com 58 e 148 árvores danificadas/ha.

A diferença entre essas áreas de colheita é que, no presente estudo, a empresa é proprietária das terras e realizou a colheita planejadamente. No maioria dos casos relatados por Veríssimo et al. (1996a, b), o madeireiro não era o proprietário das terras. Pressupõem-se que, por ser a empresa proprietária das terras, espera-se que esta seja mais consciente dos danos que pode causar ao patrimônio mais valioso de uma empresa de base florestal, isto é, a floresta, e, portanto, estará mais comprometida com a sustentabilidade da floresta. Por conseguinte, a empresa planeja e executa mais corretamente todas as atividades de pré-colheita (corte de cipós, inventário $100 \%$, com mapeamento, abertura das trilhas de arraste e direcionamento de queda), colheitas (abate, extração, carga e transporte), tratamentos silviculturais e monitoramento da floresta.

No Quadro 1 estão os resultados da análise da variância que relaciona a estrutura das florestas primárias não-explorada $(\boldsymbol{F P N E})$ e explorada $(\boldsymbol{F P E})$ com a posição de danos $(\boldsymbol{P d})$, no nível I de inclusão de $\boldsymbol{D} \boldsymbol{A P}$. Observase que houve efeito significativo $(\boldsymbol{P}<0,01)$ entre as florestas e entre as diferentes posições de danos, bem como na interação floresta versus posição de danos em todas as características discriminadas pelo teste de $\boldsymbol{F}$.

Quadro 1 - Resultado da análise de variância, em relação à estrutura das florestas primárias não-explorada (FPNE) e explorada $(\boldsymbol{F P E})$ e à posição de danos $(\boldsymbol{P d})$, no nível I de inclusão $(\boldsymbol{D A P} \geq 15,0 \mathrm{~cm})$, em que $\boldsymbol{D A}=$ densidade absoluta (n/ha), $\boldsymbol{D o A}=$ dominância absoluta $\left(\mathrm{m}^{2} / \mathrm{ha}\right)$ e $\boldsymbol{V o l}=$ volume de madeira $\left(\mathrm{m}^{3} / \mathrm{ha}\right)$

Table 1 - Analysis of variance results, in relation to the structure of non-exploited (FPNE) and exploited (FPE) old growth forests and location of damage $(\boldsymbol{P d})$ in level $I$ of approach $(\mathbf{D B H} \geq 15.0 \mathrm{~cm})$, where: $\boldsymbol{D A}=$ absolute density $(\mathrm{n} / \mathrm{ha})$; $\boldsymbol{D o A}=$ absolute dominance $\left(\mathrm{m}^{2} / \mathrm{ha}\right)$; and $\mathbf{V o l}=$ volume of $\operatorname{wood}\left(\mathrm{m}^{3} / \mathrm{ha}\right)$

\begin{tabular}{|l|c|c|c|c|}
\hline \multirow{2}{*}{ Fonte de Variação } & \multirow{2}{*}{ GL } & \multicolumn{3}{|c|}{ Quadrados Médios } \\
\cline { 3 - 5 } & & $\boldsymbol{D A}(\mathrm{n} / \mathrm{ha})$ & $\boldsymbol{D o A}\left(\mathrm{m}^{2} / \mathrm{ha}\right)$ & $\boldsymbol{V o l}\left(\mathrm{m}^{3} / \mathrm{ha}\right)$ \\
\hline Floresta & 1 & $4.014,1^{* *}$ & $28,52 * *$ & $2.164,95 * *$ \\
Posição de danos & 4 & $123.878,3^{* *}$ & $732,28^{* *}$ & $58.086,50 * *$ \\
Floresta X Posição de danos & 4 & $4.001,9 * *$ & $25,11^{* *}$ & $2.343,14 * *$ \\
\hline Resíduo & 40 & 269,3 & 2,59 & 245,07 \\
\hline Média & & 58,1 & 4,54 & 37,56 \\
\hline CVexp (\%) & & 28,2 & 35,45 & 41,68 \\
\hline
\end{tabular}

“*” e “**" = significativos a 5 e $1 \%$ de probabilidade, respectivamente, pelo teste $\mathrm{F}$.

$\mathrm{ns}=$ não-significativos a 5 e $1 \%$ de probabilidade, respectivamente, pelo teste $\mathrm{F}$. 
Diante desses resultados, o comportamento da interação florestas x posição de danos, em relação às características analisadas, pode ser observado no Quadro 2.

Quando se comparam os resultados do teste de médias referentes às florestas versus posições de danos, verifica-se (Quadro 2) que tanto na $\boldsymbol{F P N E}$ quanto na $\boldsymbol{F P E}$ predominaram, no nível I de inclusão $(\boldsymbol{D} \boldsymbol{A P} \geq 15,0 \mathrm{~cm})$, árvores sem danos $\left(\boldsymbol{P d _ { 1 }}\right)$.

No Quadro 3 estão os resultados da análise da variância que relaciona a estrutura das florestas primárias não-explorada $(\boldsymbol{F P N E})$ e explorada $(\boldsymbol{F P E})$ com a posição de danos $(\boldsymbol{P d})$, no nível II de inclusão $(5,0 \leq \boldsymbol{D} \boldsymbol{A P}$ $<15,0 \mathrm{~cm})$. Observa-se que houve efeito significativo $(\boldsymbol{P}<0,01)$ entre as diferentes posições de danos. Entretanto, não foram verificadas diferenças significativas, pelo teste de $\boldsymbol{F}$, para florestas e também para a interação floresta versus posição de danos.

Os resultados da comparação das médias entre as diferentes posições de danos, referentes às características analisadas, estão no Quadro 4.

Quadro 2 - Resultados do teste de Tukey para as médias de florestas e posição de danos (Pd) em relação às variáveis avaliadas no nível I de inclusão $(\boldsymbol{D A P} \geq 15,0 \mathrm{~cm})$, em que $\boldsymbol{P N} \boldsymbol{E}=$ floresta primária não-explorada, $\boldsymbol{F P E}=$ floresta primária explorada $\boldsymbol{P} \boldsymbol{d}_{1}=$ sem danos $\boldsymbol{P} \boldsymbol{d}_{2}=$ danos no tronco $\boldsymbol{P} \boldsymbol{d}_{3}=$ danos na copa $\boldsymbol{P d _ { 4 }}=$ danos no tronco e na copa, $\boldsymbol{P d} \boldsymbol{d}_{5}=$ árvore morta; $\boldsymbol{D A}=$ densidade absoluta $(\mathrm{n} / \mathrm{ha}) \boldsymbol{D o A}=$ dominância absoluta $\left(\mathrm{m}^{2} / \mathrm{ha}\right)$ e $\boldsymbol{V o l}=$ volume de madeira $\left(\mathrm{m}^{3} / \mathrm{ha}\right)$

Table 2 - Tukey's test results for the forest means and location of damage $(P d)$ in relation to the variables evaluated in level I of approach (DBH $\geq 15.0 \mathrm{~cm})$, where: $\boldsymbol{F P N E}=$ non-exploited forest $; \boldsymbol{F P E}=$ exploited forest $; \boldsymbol{P} \boldsymbol{d}_{1}=$ without damage; $\boldsymbol{P} \boldsymbol{d}_{2}$ $=$ damage on trunk $; \boldsymbol{P d}_{3}=$ damage on crown $; \boldsymbol{P} \boldsymbol{d}_{4}=$ damage on trunk and crown $;$ and $\boldsymbol{P} \boldsymbol{d}_{5}=$ dead tree $; \boldsymbol{D A}=$ absolute density $(\mathrm{n} / \mathrm{ha}) ; \boldsymbol{D o A}=$ absolute dominance $\left(\mathrm{m}^{2} / \mathrm{ha}\right) ;$ and $\boldsymbol{V o l}=$ volume of $\operatorname{wood}\left(\mathrm{m}^{3} / \mathrm{ha}\right)$

\begin{tabular}{|c|c|c|c|c|c|c|}
\hline \multirow{2}{*}{$\begin{array}{c}\text { Posição de } \\
\text { Danos }\end{array}$} & \multicolumn{2}{|c|}{$\boldsymbol{D} \boldsymbol{A}(\mathrm{n} / \mathrm{ha})$} & \multicolumn{2}{|c|}{$\boldsymbol{D o} \boldsymbol{A}\left(\mathrm{m}^{2} / \mathrm{ha}\right)$} & \multicolumn{2}{|c|}{ Vol $\left(\mathrm{m}^{3} / \mathrm{ha}\right)$} \\
\hline & FPNE & $F P E$ & FPNE & $F P E$ & FPNE & $F P E$ \\
\hline$P d_{1}$ & $301,8 \boldsymbol{A} a$ & $212,4 \mathrm{~A} b$ & $23,42 \mathrm{~A} a$ & $16,25 A b$ & $207,78 \mathrm{~A} a$ & $139,92 \boldsymbol{A} b$ \\
\hline$P d_{2}$ & 7,0 $\mathrm{B} a$ & $9,0 \mathrm{~B} a$ & $0,87 \boldsymbol{B} a$ & $0,79 \boldsymbol{B} a$ & $7,01 \boldsymbol{B} a$ & $5,50 \mathrm{~B} a$ \\
\hline$P d_{3}$ & $13,4 \boldsymbol{B} a$ & $13,0 \mathrm{~B} a$ & $0,71 \boldsymbol{B} a$ & $0,82 \boldsymbol{B} a$ & $4,22 \mathrm{~B} a$ & $4,58 \mathrm{~B} a$ \\
\hline$P d_{4}$ & $1,8 \mathrm{~B} a$ & $3,2 \mathrm{~B} a$ & $0,20 \mathrm{~B} a$ & $0,19 \boldsymbol{B} a$ & $0,16 \mathrm{~B} a$ & $0,73 \boldsymbol{B} a$ \\
\hline$P d_{5}$ & $11,4 \mathrm{~B} a$ & $8,2 \boldsymbol{B} a$ & $1,28 \boldsymbol{B} a$ & $0,88 \boldsymbol{B} a$ & $1,55 \mathrm{~B} a$ & $4,19 \boldsymbol{B} a$ \\
\hline
\end{tabular}

Médias seguidas de pelo menos uma mesma letra maiúscula na coluna e minúscula na linha, respectivamente, não diferem entre si pelo teste de Tukey, a 5\% de probabilidade.

Quadro 3 - Resultado da análise da variância, em relação à estrutura das florestas primárias não-explorada (FPNE) e explorada $(\boldsymbol{F P E})$ e à posição de danos $(\boldsymbol{P d})$, no nível II de inclusão $(5,0 \leq \boldsymbol{D A} \boldsymbol{P}<15,0 \mathrm{~cm})$, em que $\boldsymbol{D A}=\operatorname{densidade}$ absoluta (n/ha) $\boldsymbol{D o A}=$ dominância absoluta $\left(\mathrm{m}^{2} / \mathrm{ha}\right)$ e $\boldsymbol{V o l}=$ volume de madeira $\left(\mathrm{m}^{3} / \mathrm{ha}\right)$

Table 3 - Analysis of variance results, in relation to the structure of non-exploited (FPNE) and exploited (FPE) old growth forests and location of damage $(\boldsymbol{P d})$ in level II of approach $(5.0 \leq \mathbf{D B H}<15.0 \mathrm{~cm})$, where: $\boldsymbol{D A}=$ absolute density $(\mathrm{n} / \mathrm{ha})$; DoA = absolute dominance $\left(\mathrm{m}^{2} / \mathrm{ha}\right) ;$ and $\mathbf{V o l}=$ volume of $w o o d\left(\mathrm{~m}^{3} / \mathrm{ha}\right)$

\begin{tabular}{|l|c|c|c|c|}
\hline \multirow{2}{*}{ Fonte de Variação } & \multirow{2}{*}{ GL } & \multicolumn{3}{|c|}{ Quadrados Médios } \\
\cline { 3 - 5 } & & $\boldsymbol{D A}(\mathrm{n} / \mathrm{ha})$ & $\boldsymbol{D o} \boldsymbol{A}\left(\mathrm{m}^{2} / \mathrm{ha}\right)$ & $\boldsymbol{V o l}\left(\mathrm{m}^{3} / \mathrm{ha}\right)$ \\
\hline Floresta & 1 & $3.872,0^{\mathrm{ns}}$ & $0,22^{\mathrm{ns}}$ & $19,44^{\mathrm{ns}}$ \\
Posição de danos & 4 & $1.963 .028,0^{* *}$ & $63,99^{* *}$ & $687,02 * *$ \\
Floresta X Posição de danos & 4 & $2.852,0^{\mathrm{ns}}$ & $0,23^{\mathrm{ns}}$ & $12,75^{\mathrm{ns}}$ \\
\hline Resíduo & 40 & $6.260,0$ & 0,50 & 7,85 \\
\hline Média & & 209,6 & 1,22 & 3,90 \\
\hline CVexp (\%) & & $37,7^{*}$ & 57,96 & 71,90 \\
\hline
\end{tabular}

$* *=$ significativo a $1 \%$ de probabilidade, pelo teste $\mathrm{F}$.

ns = não-significativo. 
Quadro 4 - Médias de densidade absoluta $(\boldsymbol{D A})$, dominância absoluta $(\boldsymbol{D o A})$ e volume de madeira $(\boldsymbol{V o l})$ em relação à posição de danos, no nível II de inclusão $(5,0 \leq \boldsymbol{D A P}<15,0 \mathrm{~cm})$

Table 4 - Means of absolute density (DA), absolute dominance (DoA) and volume (Vol) in relation to location of damage (Pd), in level II of approach (5.0 $\leq$ DBH $<15.0 \mathrm{~cm}$ )

\begin{tabular}{|c|r|c|c|}
\hline \multirow{2}{*}{$\begin{array}{c}\text { Posição de } \\
\text { Danos }\end{array}$} & \multicolumn{3}{|c|}{ Médias } \\
\cline { 2 - 4 } & $\boldsymbol{D A}(\mathrm{n} / \mathrm{ha})$ & $\boldsymbol{D o} \boldsymbol{A}\left(\mathrm{m}^{2} / \mathrm{ha}\right)$ & $\boldsymbol{V o l}\left(\mathrm{m}^{3} / \mathrm{ha}\right)$ \\
\hline $\boldsymbol{P d}_{1}$ & $1.002,0 a$ & $5,74 a$ & $18,72 a$ \\
$\boldsymbol{P d}_{2}$ & $6,0 \mathrm{~b}$ & $0,10 \mathrm{~b}$ & $0,21 \mathrm{~b}$ \\
$\boldsymbol{P d}_{3}$ & $24,0 \mathrm{~b}$ & $0,17 \mathrm{~b}$ & $0,52 \mathrm{~b}$ \\
$\boldsymbol{P d}_{4}$ & $0,0 \mathrm{~b}$ & $0,00 \mathrm{~b}$ & $0,00 \mathrm{~b}$ \\
$\boldsymbol{P d}_{5}$ & $16,0 \mathrm{~b}$ & $0,07 \mathrm{~b}$ & $0,03 \mathrm{~b}$ \\
\hline
\end{tabular}

As médias seguidas de pelo menos uma mesma letra minúscula não diferem entre si, a $5 \%$ de probabilidade, pelo teste de Tukey.

Os resultados das médias de densidade absoluta $(\mathrm{n} / \mathrm{ha})$, dominância absoluta $\left(\mathrm{m}^{2} / \mathrm{ha}\right)$ e volume $\left(\mathrm{m}^{3} /\right.$ ha), considerando a posição de danos, no nível II de inclusão $(5,0 \leq \boldsymbol{D} \boldsymbol{A P}<15,0 \mathrm{~cm})$, indicam que houve predomínio de árvores sem danos $\left(\boldsymbol{P} \boldsymbol{d}_{\boldsymbol{1}}\right)$. Estes resultados mostram ainda que a colheita florestal não afetou significativamente o estoque remanescente de árvores com $5,0 \leq \boldsymbol{D} \boldsymbol{A} \boldsymbol{P}<15,0 \mathrm{~cm}$.

Os danos de colheita também foram classificados em função das causas em que estes ocorreram. Nesta análise constatou-se que 17 (7\%) árvores foram danificadas em função das operações de corte das árvores; 7 (3\%) foram danificadas pelas operações de extração das toras; e 9 (4\%) foram árvores mortas pelas operações de colheita florestal. É importante destacar que a média da área danificada pela abertura de trilhas de arraste, na colheita de madeira, foi de aproximadamente $666,47 \mathrm{~m}^{2} / \mathrm{ha}(6,6 \%)$ e que as clareiras, em média, representaram $944,8 \mathrm{~m}^{2} / \mathrm{ha}$ ( $9,4 \%$ ), ou seja, cerca de $29 \%$ das causas de danos foram oriundas das operações de colheita florestal. Jonkers (1987) destacou que a exploração seletiva não reduz somente o estoque de madeira, mas também causa alguns danos na vegetação remanescente, isto porque muitas árvores que não são exploradas morrem ou sofrem injúrias. $\mathrm{O}$ autor relatou ainda que as diferentes categorias de danos relacionadas à área basal representam um parâmetro importante para quantificar perdas de madeiras na área explorada, pois essa variável está fortemente correlacionada com o volume explorado.

Esses resultados, ainda assim, são considerados baixos, pois segundo Veríssimo (1996b) em Paragominas, no Estado do Pará, a área danificada pelas operações de colheita florestal ficou em torno de $3.800 \mathrm{~m}^{2} / \mathrm{ha}(38,0 \%)$.

Os resultados da análise de variância que relaciona a estrutura das florestas não-explorada e explorada com as causas de danos, no nível I de inclusão, podem ser observados no Quadro 5. Verifica-se que houve efeito significativo $(\mathrm{P}<0,01)$ entre as florestas e entre as diferentes causas de danos, bem como na interação floresta versus causa de danos, para todas as características analisadas pelo teste $F$.

Quadro 5 - Resultado da análise da variância, em relação à estrutura das florestas primárias não-explorada (FPNE) e explorada $(\boldsymbol{F P E})$ e à causa de danos, no nível I de inclusão $(\boldsymbol{D A P} \geq 15,0 \mathrm{~cm})$, em que $\boldsymbol{D A}=$ densidade absoluta $(\mathrm{n} / \mathrm{ha})$, $\boldsymbol{D o A}=$ dominância absoluta $\left(\mathrm{m}^{2} / \mathrm{ha}\right)$ e $\boldsymbol{V o l}=$ volume de madeira $\left(\mathrm{m}^{3} / \mathrm{ha}\right)$

Table 5 - Analysis of variance results, in relation to the struture of non-exploited (FPNE) and exploited (FPE) old growth forests and causes of damage $(\boldsymbol{C d})$, in level I of approach $(\mathbf{D B H} \geq 15.0 \mathrm{~cm})$, where: $\boldsymbol{D A}=$ absolute density; DoA = absolute dominance; and $\mathbf{V o l}=$ volume of wood $\left(\mathrm{m}^{3} / \mathrm{ha}\right)$

\begin{tabular}{|l|c|c|c|c|}
\hline \multirow{2}{*}{ Fonte de Variação } & \multirow{2}{*}{ GL } & \multicolumn{3}{|c|}{ Quadrados Médios } \\
\cline { 3 - 5 } & & $\boldsymbol{D A}(\mathrm{n} / \mathrm{ha})$ & $\boldsymbol{D o A}\left(\mathrm{m}^{2} / \mathrm{ha}\right)$ & $\boldsymbol{V o l}\left(\mathrm{m}^{3} / \mathrm{ha}\right)$ \\
\hline Floresta & 1 & $5.017,6 * *$ & $35,65 * *$ & $2.706,19 * *$ \\
Posição de danos & 3 & $150.922,5 * *$ & $890,45 * *$ & $71.576,55 * *$ \\
Floresta X Posição de danos & 3 & $5.108,3 * *$ & $33,68 * *$ & $3.004,77 * *$ \\
\hline Resíduo & 32 & 329,5 & 3,12 & 303,19 \\
\hline Média & & 72,6 & 5,68 & 46,95 \\
\hline CVexp (\%) & & 25,0 & 31,10 & 37,08 \\
\hline
\end{tabular}

$* *$ = significativo a $1 \%$ de probabilidade, pelo teste $\mathrm{F}$. 
A comparação das médias entre as florestas e as diferentes causas de danos, referentes às características analisadas, pode ser observada no Quadro 6. Os resultados foram semelhantes aos encontrados para comparação entre florestas $\mathbf{x}$ posição de danos, indicando que a causa de danos 1 (sem danos evidentes) mostrou-se diferenciada em relação às outras causas de danos. Tal fato demonstra, mais uma vez, que há predomínio de árvores sem dano nas duas florestas estudadas.

Para o nível II de inclusão $(5,0 \leq \boldsymbol{D} \boldsymbol{A P}<15,0 \mathrm{~cm})$, os resultados da análise de variância entre as florestas e as causas de danos em relação à densidade (n/ha), à dominância $\left(\mathrm{m}^{2} / \mathrm{ha}\right)$ e ao volume $\left(\mathrm{m}^{3} / \mathrm{ha}\right)$ encontram-se no Quadro 7. Observa-se que houve diferença significativa $(\mathrm{P}<0,01)$ apenas entre as diferentes causas de danos, pelo teste de F. Resultado idêntico também foi encontrado, quando se analisou o nível II de inclusão para floresta $\mathbf{x}$ posição de danos, o que indica que não existe diferença significativa entre as posições e as causas de danos 2, 3, 4, e 5 .

No nível II de inclusão (Quadro 8), os resultados da comparação das médias entre as causas de danos, em relação às características analisadas, foram semelhantes aos obtidos para posição de danos. Uma vez que $\boldsymbol{P d}_{1}$ e

Quadro 6 - Médias de densidade absoluta $(\boldsymbol{D A})$, dominância absoluta (DoA) e volume de madeira(Vol) em relação às florestas primárias não-explorada (FPNE) e explorada $(\boldsymbol{F P E})$ e à causa de danos $(\boldsymbol{C d})$, no nível I de inclusão $(\boldsymbol{D A P} \geq 15,0 \mathrm{~cm})$, em que $\boldsymbol{C} \boldsymbol{d}_{1}=$ sem danos, $\boldsymbol{C} \boldsymbol{d}_{2}=$ operação de corte, $\boldsymbol{C d}_{3}=$ operação de extração e $\boldsymbol{C} \boldsymbol{d}_{4}=$ árvore morta pelas operações de colheita florestal

Table 6 - Means of absolute density ( $\mathrm{n} / \mathrm{ha})$, absolute dominance $\left(\mathrm{m}^{2} / \mathrm{ha}\right)$ and volume of wood $\left(\mathrm{m}^{3} / \mathrm{ha}\right)$ in relation to the non-exploited (FPNE) and exploited (FPE) old growth forests and causes of damage (CD), in level I of

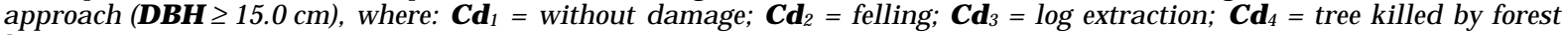
harvest activities

\begin{tabular}{|c|c|c|c|c|c|c|}
\hline \multirow{2}{*}{$\begin{array}{c}\text { Posição de } \\
\text { Danos }\end{array}$} & \multicolumn{2}{|c|}{$\boldsymbol{D} \boldsymbol{A}$ (n/ha) } & \multicolumn{2}{|c|}{$\boldsymbol{D o} \boldsymbol{A}\left(\mathrm{m}^{2} / \mathrm{ha}\right)$} & \multicolumn{2}{|c|}{$\operatorname{Vol}\left(\mathrm{m}^{3} / \mathrm{ha}\right)$} \\
\hline & FPNE & $F P E$ & FPNE & $F P E$ & FPNE & $F P E$ \\
\hline $\begin{array}{l}C d_{1} \\
C d_{2} \\
C d_{3} \\
C d_{4}\end{array}$ & $\begin{array}{r}301,2 \boldsymbol{A} a \\
14,0 \boldsymbol{B} a \\
19,6 \boldsymbol{B} a \\
0,6 \boldsymbol{B} a\end{array}$ & $\begin{array}{r}212,4 \boldsymbol{A} b \\
17,2 \boldsymbol{B} a \\
6,8 \boldsymbol{B} a \\
9,4 \boldsymbol{B} a\end{array}$ & $\begin{array}{r}23,39 \boldsymbol{A} a \\
0,87 \boldsymbol{B} a \\
2,19 \boldsymbol{B} a \\
0,03 \boldsymbol{B} a\end{array}$ & $\begin{array}{r}16,25 \boldsymbol{A} b \\
1,14 \boldsymbol{B} a \\
0,54 \boldsymbol{B} a \\
1,00 \boldsymbol{B} a\end{array}$ & $\begin{array}{r}207,77 \boldsymbol{A} a \\
4,10 \boldsymbol{B} a \\
8,84 \boldsymbol{B} a \\
0,00 \boldsymbol{B} a\end{array}$ & $\begin{array}{r}139,92 \boldsymbol{A} b \\
6,45 \boldsymbol{B} a \\
2,47 \boldsymbol{B} a \\
6,08 \boldsymbol{B} a\end{array}$ \\
\hline
\end{tabular}

Médias seguidas de pelo menos uma mesma letra maiúscula na coluna e minúscula na linha, respectivamente, não diferem entre si pelo teste de Tukey, a 5\% de probabilidade.

Quadro 7 - Resultado da análise da variância, em relação à estrutura das florestas primárias não-explorada (FPNE) e explorada $(\boldsymbol{F P E})$ e à causa de danos $(\boldsymbol{C} \boldsymbol{d})$, no nível II de inclusão $(5,0 \leq \boldsymbol{D A} \boldsymbol{P}<15,0 \mathrm{~cm})$, em que $\boldsymbol{D A}=$ densidade absoluta (n/ha), $\boldsymbol{D o A}=$ dominância absoluta $\left(\mathrm{m}^{2} / \mathrm{ha}\right)$ e $\boldsymbol{V o l}=$ volume de madeira $\left(\mathrm{m}^{3} / \mathrm{ha}\right)$

Table 7 - Variance analysis results, in relation to the structure of non-exploited (FPNE) and exploited (FPE) old growth forests and causes of damage $(\boldsymbol{C d})$, in level II of approach $(5.0 \leq \mathbf{D B H}<15.0 \mathrm{~cm})$, where: $\boldsymbol{D A}=$ absolute density; DoA = absolute dominance; and $\mathbf{V o l}=$ volume of wood

\begin{tabular}{|l|c|c|c|c|}
\hline \multirow{2}{*}{ Fonte de Variação } & \multirow{2}{*}{ GL } & \multicolumn{3}{|c|}{ Quadrados Médios } \\
\cline { 3 - 5 } & & $\boldsymbol{D A}(\mathrm{n} / \mathrm{ha})$ & $\boldsymbol{D o} \boldsymbol{A}\left(\mathrm{m}^{2} / \mathrm{ha}\right)$ & $\boldsymbol{V} \boldsymbol{l}\left(\mathrm{m}^{3} / \mathrm{ha}\right)$ \\
\hline Floresta & 1 & $3.610,0^{\mathrm{ns}}$ & $0,31^{\mathrm{ns}}$ & $24,05^{\mathrm{ns}}$ \\
Posição de danos & 3 & $2.397 .983,0^{* *}$ & $77,53^{* *}$ & $831,58^{* *}$ \\
Floresta X Posição de danos & 3 & $4.836,7^{\mathrm{ns}}$ & $0,36^{\mathrm{ns}}$ & $18,47^{\mathrm{ns}}$ \\
\hline Resíduo & 32 & $8.019,9$ & 0,64 & 10,14 \\
\hline Média & & 263,5 & 1,52 & 4,87 \\
\hline CVexp (\%) & & 34,0 & 52,33 & 65,45 \\
\hline
\end{tabular}

$* *=$ significativo a $1 \%$ de probabilidade, pelo teste $\mathrm{F}$.

$\mathrm{ns}=$ não-significativo. 
$\boldsymbol{C d}_{1}$ têm o mesmo significado, esta análise ratificou a supremacia das árvores sem danos. Dentre as árvores danificadas, sejam elas sobreviventes ou mortas, verificou-se que não houve efeitos significativos das operações de corte e das operações de extração.

Quadro 8 - Médias da densidade absoluta (DA), dominância absoluta $(\boldsymbol{D o A})$ e volume de madeira $(\boldsymbol{V o l})$ em relação às florestas primárias não-explorada $(\boldsymbol{F P N E})$ e explorada (FPE) e à causa de danos (Cd), no nível II de inclusão $(5,0 \leq \boldsymbol{D A P}<15,0 \mathrm{~cm})$

Table 8 - Means of absolute density (DA), absolute dominance $(D o A)$ and volume of wood $(\boldsymbol{V o l})$ in relation to non-exploited (FPNE) and exploited (FPE) old growth forests and causes of damage (Cd), in level II of approach $(5.0 \leq \mathbf{D B H}<15.0 \mathrm{~cm})$

\begin{tabular}{|c|c|c|c|}
\hline Posição de & \multicolumn{3}{|c|}{ Médias } \\
\cline { 2 - 4 } Danos & $\boldsymbol{D A}(\mathrm{n} / \mathrm{ha})$ & $\boldsymbol{D o} \boldsymbol{A}\left(\mathrm{m}^{2} / \mathrm{ha}\right)$ & $\boldsymbol{V o l}\left(\mathrm{m}^{3} / \mathrm{ha}\right)$ \\
\hline $\boldsymbol{C d}_{1}$ & $998,00 a$ & $5,70 a$ & $18,54 a$ \\
$\boldsymbol{C d}_{2}$ & $24,00 b$ & $0,14 b$ & $0,34 b$ \\
$\boldsymbol{C d}_{3}$ & $20,00 b$ & $0,15 b$ & $0,11 b$ \\
$\boldsymbol{C d}_{4}$ & $12,00 b$ & $0,11 b$ & $0,48 b$ \\
$\boldsymbol{C d}_{1}$ & $998,00 a$ & $5,70 a$ & $18,54 a$ \\
\hline
\end{tabular}

As médias seguidas de pelo menos uma mesma letra minúscula não diferem entre si a $5 \%$ de probabilidade, pelo teste de Tukey.

\section{CONCLUSÕES}

- A exploração seletiva de madeira não provocou danos significativos nas árvores, nos níveis I $(\boldsymbol{D} \boldsymbol{A P} \geq 15 \mathrm{~cm})$ e II $(5 \leq \boldsymbol{D} \boldsymbol{A P}<15 \mathrm{~cm})$ de inclusão.

- As modificações nas estruturas de diâmetro, área basal e volume da floresta primária explorada foram mais evidentes nas maiores classes diamétricas, principalmente das espécies comerciais.

- Os danos provocados pela colheita na floresta primária foram baixos, apesar da redução de $\boldsymbol{D A}, \boldsymbol{D o} \boldsymbol{A}$ e $\boldsymbol{V o l}$ na floresta primária explorada.

- A colheita florestal realizada e executada de acordo com critérios técnicos, dentro dos princípios do manejo florestal sustentável, pode minimizar os danos às árvores remanescentes e garantir a sustentabilidade da floresta.

\section{REFERÊNCIAS BIBLIOGRÁFICAS}

BRASIL. Ministério das Minas e Energia Departamento Nacional da Produção Mineral. Projeto RadamBrasil; folha SB. 20 - Purus. Rio de Janeiro: DNPM, 1978. 566 p. (Levantamento de recursos naturais; v.17).

R. Árvore, Viçosa-MG, v.26, n. 4, p.459-466, 2002
GREENPEACE Face a face com a destruição: relatório Greenpeace sobre as companhias multinacionais madeireiras na Amazônia Brasileira. São Paulo: 1999. 21 p.

HENDRISON, J. Damage-controlled logging in managed tropical rain forest in Suriname. Wageningen:

Agricultural University, 1989. 204 p.

FUNDAÇÃO INSTITUTO BRASILEIRO DE GEOGRAFIA E ESTATÍSTICA - IBGE. Mapa da vegetação do Brasil. Brasília: 1993. 1 p.

JONKERS, W. B. J. Vegetation structure, logging damage and silviculture in a tropical rain Forest in Suriname. Wageningen: Agricultural University, 1987. 172 p.

LANLY, J. P. Tropical forest resources. Rome: FAO, 1982. (FAO Forestry paper, 30).

OTTO, B. F. Enciclopédia da Amazônia Brasileira Manaus: Pracco, 1996. v. 1.

MINETTE, L. J. Análise de fatores operacionais e ergonômicos na operação de corte florestal com motosserra. Viçosa, MG: Universidade Federal de Viçosa, 1996. 211 p. Tese (Doutorado em Ciência Florestal) Universidade Federal de Viçosa, 1996.

SILVA, J. N. M.; LOPES, J. C. A. Inventário florestal contínuo em florestas tropicais: a metodologia utilizada pela EMBRAPA-CPATU na Amazônia Brasileira. Belém: EMBRAPA, 1984. $36 \mathrm{p}$.

UHL, C. et al. Uma inclusão integrada de pesquisa sobre o manejo dos recursos naturais na Amazônia. In: A

EXPANSÃO da atividade madeireira na amazônia: impactos e perspectivas para o desenvolvimento do setor florestal do pará. Belém: IMAZON, 1996. p. 143-163.

VERÍSSIMO, A. et al. Impactos sociais, econômicos e ecológicos da exploração seletiva de madeiras numa região de fronteira na Amazônia oriental: o caso de Tailândia. In: A EXPANSÃO da atividade madeireira na amazônia: impactos e perspectivas para o desenvolvimento do setor florestal do pará. Belém: IMAZON, 1996a. p. 09-43.

VERÍSSIMO, A. et al. Impactos da atividade madeireira e perspectivas para o manejo sustentável numa velha fronteira da Amazônia: o caso de Paragominas. In: A EXPANSÃO da atividade madeireira na amazônia: impactos e perspectivas para o desenvolvimento do setor florestal do pará. Belém: IMAZON, 1996b. p. 47-73. 